\title{
Defragmenting the Cloud Using Demand-based Resource Allocation
}

\begin{abstract}
Public clouds sell capacity in the form of pre-defined virtual machine (VM) configurations to their tenants. This forces tenants to buy the VM configuration based on the peak usage. This diminishes the value proposition of moving to a public cloud as compared to doing consolidation in a private virtualized datacenter. Ideally we would like the cloud tenants to buy capacity in bulk and benefit from statistical multiplexing among workloads. This requires dynamic allocation of bulk capacity among VMs of a tenant that may be running on different servers across different datacenters.

In this paper, we propose two novel algorithms called BPX and IDD that are able to provide the abstraction of buying bulk capacity to a cloud customer. These algorithms dynamically allocate the overall capacity between VMs based on their demand and user-set importance. Both algorithms are highly scalable and are designed to work in a large scale environment. Our analysis shows that BPX is able to meet all the desirable properties in providing the abstraction. We implemented the prototype of BPX as part of VMware's management software and showed that BPX is able to closely mimic the behavior of a centralized allocator, in a distributed manner.
\end{abstract}

\section{Introduction}

Consider an IT department of a small company that is considering moving its workloads to a public cloud. Currently the company is running a private cloud, where they are able to take advantage of server consolidation by using in-house virtualization and cloud management software. Several companies offer solutions in this space, such as Nebula [16], Nimbula [17], VMware vCloud Suite [27], Microsoft hyper$\mathrm{V}[15]$ and others.

Within the private cloud the VMs run in a controlled physical infrastructure maintained by the IT department of the same company. The private cloud is able to exploit temporal variations in the VM loads to reduce the amount of provisioned resources, by over-committing server CPU and memory. Hypervisors such as VMware ESX Server provide several techniques (like transparent page-sharing, ballooning, compression, and swap-to-SSD) to facilitate high consolidation ratios. The gains from statistical multiplexing benefit the bottom line of the company by reducing both its capital and operating expenses. In a public cloud the physical infrastructure is distributed over one or more mega data centers, supporting thousands of servers and hosting VMs belonging to multiple paying customers. In this situation the benefits of workload multiplexing accrue to the cloud service provider and not directly to the tenant, as the former increases consolidation ratios without regard to specific customers.

In the public cloud, the tenant's is forced to purchase

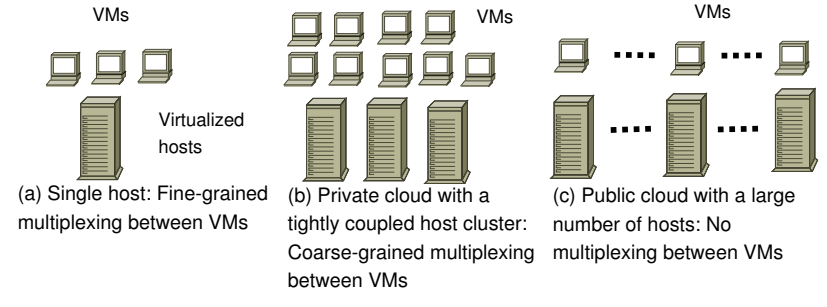

Figure 1. Hosting of VMs at different scales. As scale increases, the benefit from statistical multiplexing diminishes

VMs based on their configured sizes. Typically VMs are configured for peak usage and the consolidation helps because VMs can use resource from each other based on their run-time demands on the host. An interesting study [12] on buying capacity in terms of fixed T-shirt sizes vs. doing time sharing showed that buying per VM capacity can be twice as expensive as compared to having a time sharing system.

Lets assume that the company's private datacenter has 100 physical servers, each with $16 \mathrm{GHz}$ compute capacity and 96 GB of RAM, for a total installed capacity of 1600 $\mathrm{GHz}$ of CPU and $9600 \mathrm{~GB}$ of RAM. By exploiting multiplexing techniques, a single server may host VMs with total virtual CPU and memory capacity that add up to $32 \mathrm{GHz}$ and 192 GB respectively, providing a 2-to-1 over-commitment. Studies have noted that this ratio is typically higher for environments like virtual desktops [3, 19] and lower for enterprise workloads like Exchange and Databases.

When moving to a public cloud the company loses the advantages of over-commitment, and will have to buy resources equivalent to the size of all its VMs. This comes to the total capacity of 200 physical servers, even though total demand at any instant is half of this amount. The benefits of the statistical multiplexing are enjoyed by the cloud service provider, who can consolidate more VMs based on actual resource consumptions instead of VM's configured size.

Figure 1 shows a comparison between three different models of running VMs: (a) on a single host, (b) on a tightly-coupled cluster of hosts in a private cloud, or (c) on a loosely-coupled ensemble of hosts in a public cloud. The models differ in the mechanisms used for resource multiplexing and the time-scales at which they operate: for example, hundreds of milliseconds for a host using a scheduler within hypervisor, and minutes for a private cloud using solutions like VMware DRS [13].

None of the public clouds offer capability of resource flow between the VMs of a tenant at large scale. Doing this dynamic resource flow at the scale of the public cloud is difficult. VMware vSphere provides such an abstraction within a small scale of up to 32 hosts using features like DRS [13] (Distributed Resource Manager). DRS monitors per host utilization every five minutes and performs loadbalancing across hosts using live migration, and also adjusts 


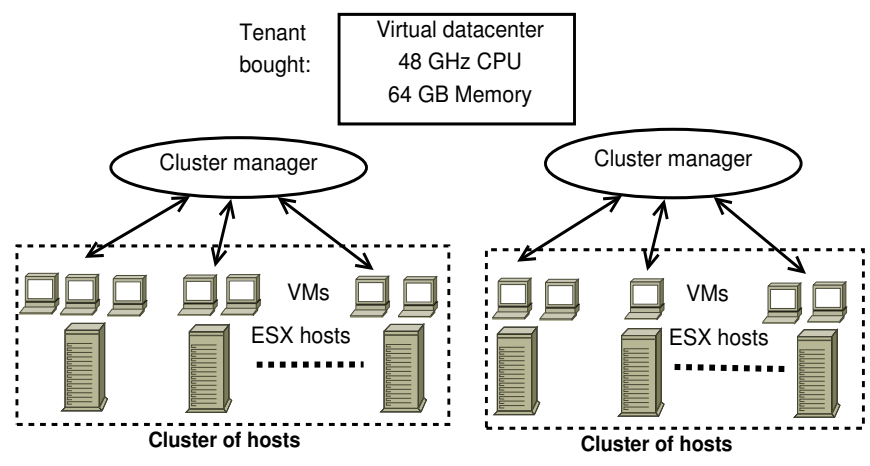

Figure 2. System overview and deployment in a cloud

resource controls to provide more resources to VMs with higher demand.

In this paper we present a model for buying bulk shareable capacity in the cloud similar to that provided by a dedicated physical datacenter. The tenant can flexibly multiplex this purchased capacity dynamically among its VMs. To realize that model we present two distributed resource allocation algorithms that periodically re-distribute the purchased capacity among the tenant's VMs based on their demand, and other tenant-specified controls such as reservations, limits and priority (or shares). This is challenging because a tenant's VMs may be spread across an arbitrary number of physically distributed servers, and because of the need to handle tens of thousands of such customers in a public cloud. We highlight these challenges using an example in Section 2.

The distributed allocation algorithms called IDD (Iterative Distributed Divvy) and BPX (Base + Proportional Excess) are described in Section 3. The two algorithms make different choices in the relative importance of VMs dynamic demands versus their static priorities, while adhering to basic allocation constraints. The allocation of IDD matches that of the centralized VMware DRS resource manager. However, unlike DRS that is centralized and limited to a small number of servers, IDD can work in a distributed environment. BPX is a fully-asynchronous distributed algorithm that avoids some thorny real-world implementation issues of IDD, thus making it more robust and scalable. Due to its better properties we did extensive experimental evaluation only of BPX.

We implemented BPX as a prototype in VMware's management software. Our evaluation (Section 5) demonstrates that BPX is able to allocate more CPU and memory resources to VMs as compared to a static allocation when the workload demands fluctuate. Also BPX is able to closely match the allocations that would be made by IDD, without the implementation problems of the latter. We finally conclude with some directions for future work in Section 7.

\section{System Model and Allocation Policies}

We begin by looking at the physical structure of a cloud environment since it places constraints on the practicality of resource allocation mechanisms. Figure 2 shows an overview of a typical virtualized datacenter used for a public cloud environment. Tenants buy a certain capacity in the form of

\begin{tabular}{|l|l|l|l|}
\hline Buying model & $\begin{array}{l}\text { Capacity } \\
\text { Needed }\end{array}$ & Cost & $\begin{array}{l}\text { Statistical multi- } \\
\text { plexing }\end{array}$ \\
\hline \hline VM's configured size & $48 \mathrm{GHz}$ & High & Not-needed \\
\hline VM's demand & $20 \mathrm{GHz}$ & Low & No \\
\hline $\begin{array}{l}\text { Total demand with dy- } \\
\text { namic allocation }\end{array}$ & $20 \mathrm{GHz}$ & Low & Yes \\
\hline
\end{tabular}

Table 1. Comparison between various buying models

a virtual datacenter (VDC). Tenants then deploy VMs based on the capacity available in a VDC in a self-service manner. These VMs are deployed on top of the physically distributed infrastructure.

The physical infrastructure is divided into a large pool of compute and storage servers. The former are organized into clusters consisting of tens of servers (typically 32 or so). A public cloud may contain hundreds of such clusters to get a large-scale deployment. The VMs from a single tenant may span an arbitrary set of clusters. This architecture exists for most of the deployments based on solutions from VMware vSphere [27], Microsoft SCVMM [15] and others.

In this environment it is infeasible to simply extend currently existing resource allocation mechanisms. The stateof-art today includes cluster management solutions like DRS [13] that collect information about VMs from each server in the cluster, and allocate CPU and memory resources based on the demand. This clustered model has certain advantages like facilitating VM migrations between servers if the total allocation to VMs on a server exceeds its physical capacity. However, when a tenant's VMs are spread across multiple clusters, a centralized strategy becomes impractical, since it requires dynamic per VM information to be made available at a cloud-level database shared among hundreds of clusters.

Not only does this require a massive amount of information to be frequently exchanged between clusters, but the centralized algorithms will be CPU intensive due to the large number of VMs it needs to consider. This scalable dynamic resource flow problem is difficult, and we are not aware of any practical existing solution. We envision our algorithm to run at the cluster-level and allow distributed clusters to work together to provide the customer with the abstraction of buying bulk capacity. One can also run our solution at the server level, but higher-level monitoring would still be needed to balance server loads and initiate migrations from overloaded servers.

Example 1: We use an example to motivate the need for the bulk capacity abstraction and the desired properties of a solution. Consider a customer who wants to run eight VMs in the cloud. Four of them (called $H_{1}, H_{2}, H_{3}$ and $H_{4}$ ) are running a production application with high priority, while the other four VMs (called $L_{1}, L_{2}, L_{3}$ and $L_{4}$ ) are running an internal application of lower priority. Each high-priority VM can spike up to $8 \mathrm{GHz}$ at different times, but their total workload requirements (determined by application profiling and past experience) is within $16 \mathrm{GHz}$ most of the time. Similarly the low-priority VMs can peak up to $4 \mathrm{GHz}$ individually, but the customer may only want to allocate a total of 4 $\mathrm{GHz}$ combined to all four of them.

Such usage patterns are fairly common in an IT depart- 
ment due to presence of a wide variety of workloads, and demand fluctuations caused by diurnal cycles. The VMs may be running in different servers on different racks, as determined by the cloud provider's software and VM placement policies. A key question is how much capacity should the customer buy from a public cloud to support such a use case? There are three possible solutions:

I. Buy configured sizes for all VMs: The customer will buy the configured size (based on peak demand) for each VM, which is $8 \mathrm{GHz}$ for each of the production VMs and $4 \mathrm{GHz}$ for each of the low priority VMs. In total the customer will have to buy $8 \times 4+4 \times 4=48 \mathrm{GHz}$, of CPU resources. This solution will always meet the needs of the VMs without further intervention, but at a significantly higher cost.

II. Buy based on average VM demand: In this case, the customer will only buy the typical demand for each high priority VM, and a fixed capacity for the low priority VMs. This will be $4 \mathrm{GHz}$ each for the former and $1 \mathrm{GHz}$ each for the rest, for a total of $20 \mathrm{GHz}$. This solution although costeffective, doesn't allow sharing of resources between VMs during spikes in demand.

III. Buy aggregated demand with dynamic resource allocation: In this case, the customer will still buy $20 \mathrm{GHz}$ total capacity. However, the cloud resource management system will dynamically allocate this amount among VMs based on their actual demand. Customers can also distinguish between VMs by using additional VM-level resource controls, such as shares (or weights), minimum reservation, and maximum limit, which are incorporated into the allocation mechanisms. Table 1 shows the three buying models and a summary of their properties.

\subsection{Allocation Requirements}

We begin with a simple model where each VM $i$ is characterized by two parameters: a share value $s(i)$ that reflects its priority relative to other VMs of the customer; and a demand value $d(i)$ that reflects the predicted demand of this $\mathrm{VM}$ in the immediate future. Other controls like VM reservations and limits can be added to the model quite straightforwardly, and the details are presented later in Section 4. The demand may be based on a simple time-averaged window of actual past usage or can employ more sophisticated prediction schemes that provides margin for unexpected bursts (see Section 4). The exact prediction algorithm is an orthogonal issue to the allocation model. The share is a static parameter set by the customer at the time of VM creation, while the demand is estimated periodically by the run-time software.

The customer buys a certain aggregate amount of capacity $\mathcal{C}$. The goal of the resource manager is to allocate $\mathcal{C}$ among the VMs in an equitable manner taking into account shares and not giving the VMs more than what they demand. An allocation scheme must satisfy certain sanity properties described below.

A. Location Obliviousness: Any two VMs with the same share value and the same demand should receive the same allocation independent of their location. Thus two identical workloads will make forward progress at the same rate, independent of the allocation algorithm or its local ecosystem. B. Harmonious Allocation: A VM should not be allocated more than its demand if any VM has not received its demand. This requirement ensures that there is no wasted capacity, where resources are allocated to a VM that does not currently need it, while a sibling VM gets less than its current requirements.

C. Demand-Anomaly Freedom: If two VMs have the same share value then the VM with the smaller demand should not be allocated more capacity than the one with the higher demand.

D. Share-Anomaly Freedom: If two VMs have the same demand then the VM with the smaller share should not receive more allocation than the one with the larger share, unless all VMs have received their demands. This is needed if a cloud providers needs to use share as part of billing.

\subsection{Allocation Policies}

In this section we describe three allocation policies, one using shares alone and two that use both demands and shares. We use these to characterize and compare various allocation algorithms.

Static Allocation Policy (SAP): A simple allocation scheme is to divide $\mathcal{C}$ among VMs in proportion to their share values. The total value of the shares of all the active VMs of the tenant is tracked. The capacity per share (denoted by $\rho$ ) is computed by dividing $\mathcal{C}$ by the total number of outstanding shares. The allocation of each VM is obtained by multiplying the capacity-per-share with its share value.

Formally, let $\mathcal{A}$ denote the set of active VMs of the customer. The capacity-per-share $\rho=\mathcal{C} / \sum_{j \in \mathcal{A}} s(j)$. The allocation of $\operatorname{VM}(i)$ is $a(i)=\rho \times s(i)$. It can be seen that the above policy satisfies properties A, C and D above, but fails to satisfy harmonious allocation property. Since the allocations are independent of demands, the policy may wastefully allocate capacity to high-share VMs with low demand, at the expense of lower share VMs with unsatisfied demand.

Dynamic Distributed Allocation Policy 1 (DDAP1): In this policy a VM's demand is an upper bound on the capacity allocated to it. Like in SAP, we first allocate capacity in proportion to the share values. However, if the allocation made to a VM exceeds its demand, its allocation is capped at its demand, and the unused capacity reallocated to other VMs with unsatisfied demand.

This model can be precisely formulated as a constrained resource-allocation problem using the capacity-per-share parameter $\rho$. Assume the total capacity to be allocated is less than the total demand; i.e. $\mathcal{C} \leq \sum_{i \in \mathcal{A}} d(i)$. We need to find $\rho^{*}$ such that the allocation $a(i)=\min \left\{d(i), \rho^{*} \times s(i)\right\}$ and $\sum_{i \in \mathcal{A}} a(i)=\mathcal{C}$. If the total capacity consistently exceeds the sum of demands, it indicates that the system has probably been over-provisioned. In this case, the excess can be distributed to the VMs in proportion to their shares.

The allocation can be seen to satisfy all the desired properties A through D. Notice that two VMs with the same share may receive the same allocation even if one's demand is much higher than the other. However, it would never result in a demand anomaly.

Dynamic Distributed Allocation Policy 2 (DDAP2) : Like DDAP1, this policy also first allocates capacity on the basis of shares and caps it at the VM's demand. However, it dif- 


\begin{tabular}{|c|c|c|c|c|c|}
\hline VM Parameters & $H_{1}$ & $H_{2}$ & $L_{1}$ & $L_{2}$ & Total \\
\hline \hline Demand & $1 \mathrm{GHz}$ & $8 \mathrm{GHz}$ & $1 \mathrm{GHz}$ & $3 \mathrm{GHz}$ & $13 \mathrm{GHz}$ \\
\hline \hline Shares & 400 & 400 & 100 & 100 & 1000 \\
\hline \hline Allocation model & $H_{1}$ & $H_{2}$ & $L_{1}$ & $L_{2}$ & Wastage \\
\hline \hline SAP & $4 \mathrm{GHz}$ & $4 \mathrm{GHz}$ & $1 \mathrm{GHz}$ & $1 \mathrm{GHz}$ & yes \\
\hline DDAP1 & $1 \mathrm{GHz}$ & $6.4 \mathrm{GHz}$ & $1 \mathrm{GHz}$ & $1.6 \mathrm{GHz}$ & no \\
\hline DDAP2 & $1 \mathrm{GHz}$ & $6 \mathrm{GHz}$ & $1 \mathrm{GHz}$ & $2 \mathrm{GHz}$ & no \\
\hline
\end{tabular}

Table 2. Comparison between various allocation Models. Total capacity of $10 \mathrm{GHz}$ is distributed among VMs.

fers in how it handles the excess capacity. DDAP2 allocates it to the VMs in the ratio of their unmet demands. That is, we allocate a portion of the capacity in proportion to the shares, and the remaining in proportion to unsatisfied demand. The details of this policy are described in Section 3.2. The policy satisfies all the desired allocation properties and the allocation algorithm has significant implementation advantages over the others in a distributed environment.

We illustrate the allocation policies using four of the VMs of Example 1. Suppose the shares of $H_{1}$ and $H_{2}$ are each 400 and their demands are 1 and $8 \mathrm{GHz}$ respectively. Similarly, suppose that $L_{1}$ and $L_{2}$ have shares of 100 and demands of 1 and $3 \mathrm{GHz}$ respectively. Table 2 shows how the three policies will allocate a capacity of $10 \mathrm{GHz}$. SAP simply allocates it in the ratio of the shares. As can be seen, $H_{1}$ is allocated $4 \mathrm{GHz}$, which is more than its demand (1 GHz), wasting capacity that VMs $H_{2}$ and $L_{2}$ could have used.

DDAP1 and DDAP2 also initially allocate capacity in the ratio of the shares. However, since they cap the allocation at the demand, $H_{1}$ and $L_{1}$ get only $1 \mathrm{GHz}$ each, leaving the excess amount $3 \mathrm{GHz}$ to be further allocated to $H_{2}$ and $L_{2}$. In DDAP1, this is assigned in the ratio of shares, resulting in additional allocations of $4 / 5 \times 3=2.4 \mathrm{GHz}$ and $1 / 5 \times 3=$ $0.6 \mathrm{GHz}$ respectively to $H_{2}$ and $L_{2}$, which is added to their initial share-based allocation. Since these do not exceed their demands the allocation is complete. Otherwise, the process is iteratively repeated using the excess capacity generated by the newly demand-capped VMs. In DDAP2, the excess capacity of $3 \mathrm{GHz}$ is allocated to $H_{2}$ and $L_{2}$ in the ratio of unmet demands, which are $4 \mathrm{GHz}$ and $2 \mathrm{GHz}$ respectively This results in additional allocations of $2 \mathrm{GHz}$ and $1 \mathrm{GHz}$ for the two VMs. One can show (see Section 3.2) that the total allocation will not exceed the demands of any of the VMs, so no additional iterations are ever needed. The allocation also satisfies all the desired properties A through D.

In the next section, we describe the IDD and BPX algorithms for implementing the dynamic demand policies in a scalable manner.

\section{Resource Allocation Algorithms}

In this section we describe two distributed allocation algorithms, Iterative Distributed Divvy (IDD) and Base plus Proportional Excess (BPX) that implement the DDAP1 and DDAP2 allocation policies respectively.

\subsection{Iterative Distributed Divvy Algorithm}

Recall, that the DDAP1 policy requires one to find a value $\rho^{*}$ for the capacity per share, such that $a(i)=\min \left\{d(i), \rho^{*} \times\right.$ $s(i)\}$ and $\sum_{i \in \mathcal{A}} a(i)=\mathcal{C}$. For a given value of $\rho, \mathrm{a}(i)$ is either capped at its demand $d(i)$ or equals $\rho \times s(i)$. In the first case we say that the VM is demand limited for $\rho$. Let $\mathcal{D}_{\rho}$ denote the set of demand-limited VMs and $\mathcal{N}_{\rho}$ the remaining VMs. For a given $\rho$, the total allocation made to all the VMs is given by $\mathcal{A}(\rho)=\sum_{i \in \mathcal{D}_{\rho}} d(i)+\rho \sum_{i \in \mathcal{N}_{\rho}} s(i)$. In Lemma 1 below we show that $\mathcal{A}(\rho)$ is a monotonically increasing function of $\rho$. This allows us to design an efficient sequential search strategy to find $\rho^{*}$ in a centralized setting, and a binary-search like strategy in a distributed setting.

LEMMA 1. If $\mathcal{N}_{\rho_{1}} \neq \emptyset$ then $\rho_{2}>\rho_{1}$ implies that $\mathcal{A}\left(\rho_{2}\right)>$ $\mathcal{A}\left(\rho_{1}\right)$.

Proof: Consider any $j \in \mathcal{N}_{\rho_{1}}$. If $j \in \mathcal{N}_{\rho_{2}}$ then its allocation $\rho_{2} \times d(j)$ is clearly greater than its allocation $\rho_{1} \times d(j)$ under $\rho_{1}$. Otherwise $j$ is demand-limited under $\rho_{2}$ and its allocation is $d(j)$; since $j \in \mathcal{N}_{\rho_{1}}$ its allocation under $\rho_{1}$ was less than $d(j)$. Hence for all $j \in \mathcal{N}_{\rho_{1}}$ the allocation under $\rho_{2}$ exceeds that under $\rho_{1}$. To complete the proof, note that if $j \in \mathcal{D}_{\rho_{1}}$ then $j$ is also in $\mathcal{D}_{\rho_{2}}$, and its allocation remains unchanged at $d(j)$.

Define the normalized demand of $\operatorname{VM}(i)$ as $u(i)=$ $d(i) / s(i)$, and reindex the VMs so that the $u_{i}$ are in nondecreasing order. A centralized algorithm searches for $\rho^{*}$ by trying out successive values of $\rho=u_{i}$ in increasing order of $u_{i}$, till it finds a pair of successive indexes $k, k+1$, such that $\mathcal{A}_{\rho}\left(u_{k}\right)<\mathcal{C} \leq \mathcal{A}_{\rho}\left(u_{k+1}\right)$. VMs with index $1 \cdots k$ are demand limited and will receive their demand; $\rho^{*}$ is obtained by dividing the remaining capacity by the total number of shares of the non demand-limited VMs. That is $\rho^{*}=\left(\mathcal{C}-\sum_{i=1}^{k} d(i)\right) / \sum_{i=k+1}^{n} s(i)$. The centralized algorithm requires the sorted list of all VMs in order of normalized demands, and requires $O(n)$ iterations. Hence, it is not a good solution in a distributed environemnt.

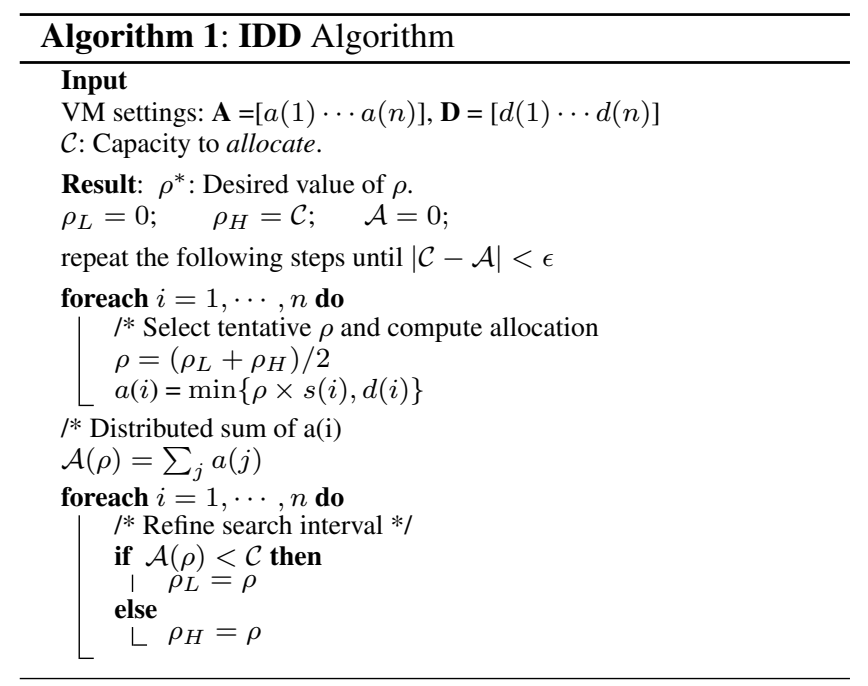

A distributed algorithm to determine $\rho^{*}$ based on a binary search strategy is shown in Algorithm 1. At the start of an iteration the value of $\rho^{*}$ will have been narrowed to lie within an interval $\left[\rho_{L}, \rho_{H}\right]$ that is known to all VMs. Each 
VM chooses a probe value of $\rho$ in the interval (for instance the mid-point), and then computes the allocation $a(i)$ based on this assumed value of $\rho$. These $\mathrm{a}(i)$ are added together in a distributed manner and the sum $\mathcal{A}(\rho)$ is returned to all VMs. The sum is compared with the actual amount of capacity $\mathcal{C}$ to be allocated. If $\mathcal{C}>\mathcal{A}(\rho)$ then by Lemma 1 the value of $\rho$ needs to be increased to make the total allocation equal to $\mathcal{C}$, and the search interval is narrowed to $\left[\rho, \rho_{H}\right]$; else the search interval is $\left[\rho_{L}, \rho\right]$. The process continues till $|\mathcal{C}-\mathcal{A}(\rho)|<\epsilon$ where $\epsilon$ is an acceptably small margin. The algorithm will converge in $O\left(\log _{2}(\mathcal{C} / \epsilon)\right)$ rounds. As described above, in each round the distributed value of the allocations need to be added up and the sum broadcast to all VMs. The number of rounds can be decreased by doing a multi-way search rather than a binary search, at the expense of communicating more information between the VMs in the addition phase. For instance, at each round the VMs could use $k>1$ probe values of $\rho, \rho_{1}, \rho_{2}, \cdots \rho_{k}$, to narrow the search interval even further in each round of communication. The allocations for each of these $\rho_{i}$ values are computed and summed independently. The next search interval is $\left[\rho_{i}, \rho_{i+1}\right]$ where $\mathcal{A}\left(\rho_{i}\right) \leq \mathcal{C}$ and $\mathcal{A}\left(\rho_{i+1}\right)>\mathcal{C}$.

Although the distributed algorithm uses only a logarithmic number of rounds (compared to linear in the number of VMs of a sequential search strategy), it has practical drawbacks. Specifically, the communication has to be synchronous. Only after all the allocations of all the nodes have been summed for a particular search range, can the next range be chosen. Having multiple synchronous rounds is slow and vulnerable to delays and failures of the underlying VMs, hosts, and communication network. Also, each tenant is periodically invoking IDD requiring the system to support multiple concurrent persistent communication sessions.

\subsection{BPX Distributed Divvy Algorithm}

In this section we describe a distributed algorithm that implements the DDAP2 policy. The solution uses a core algorithm called basicBPX shown in Algorithm 2. This routine will also be the core component in the general solution that considers other VM controls like reservations and limits.

In basicBPX Each $\mathrm{VM}(i)$ is characterized by two parameters: a lower bound on its allocation $\lambda(i)$ and an upper bound $\mu(i)$. The capacity $\mathcal{C}$ to be allocated is assumed to be at least the sum of the lower bounds. The routine first allocates each VM its lower bound $\lambda(i)$. It then divides the remaining capacity among the VMs in the ratio of $(\mu(i)-\lambda(i))$ denoted as $\delta(i)$. In a distributed setting two global sums need to be computed and retuned to the VMs: the sum of individual $\lambda(i)$ and the sum of the individual $\delta(i)$.

We use basicBPX to implement the DDAP2 allocation policy. We first divide the capacity among the VMs in proportion to their shares. We call this the fair share of the VM. VMs whose demand is less than their fair share are demandlimited, and their allocation is capped at their demand. The remaining VMs will be allocated additional capacity over their fair share. This will be done in the ratio of their unmet demand i.e. the difference between their fair share and demand.

Algorithm 3 describes the allocation algorithm formally.

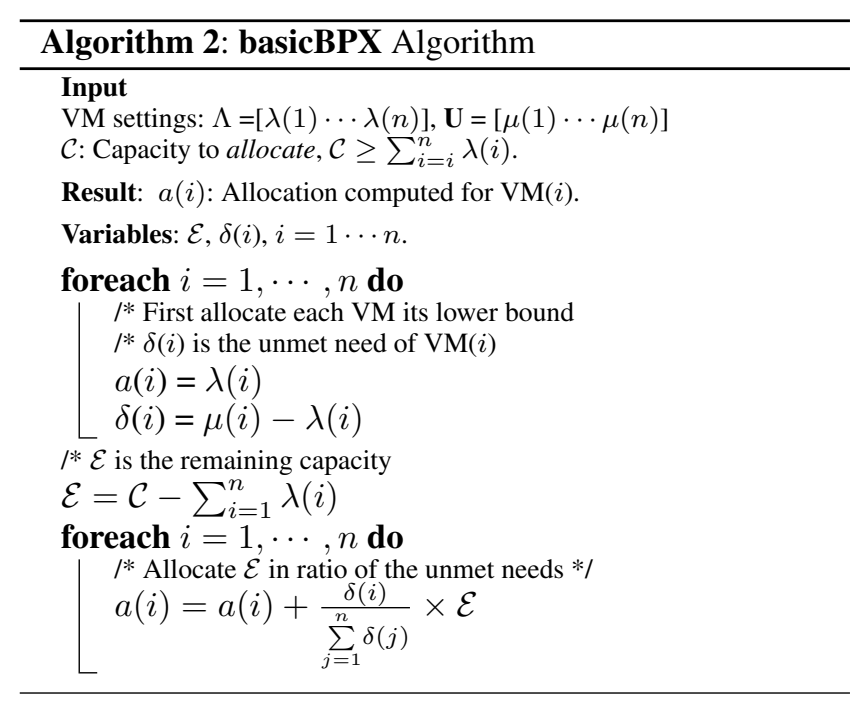

We call this algorithm as shareBPX. A VMs fair share $f(i)$ is computed by dividing the capacity $\mathcal{C}$ in proportion to the shares. Define the entitlement $e(i)$ to be the smaller of the demand and fair share of $\operatorname{VM}(i): e(i)=\min \{f(i), d(i)\}$. We then invoke basicBPX with the vector of entitlements $\mathbf{E}=[e(1) \cdots e(n)]$ as the lower-bound and the vector of demands $\mathbf{D}=[d(1) \cdots d(n)]$ as the upper bound; basicBPX first gives each VM its lower bound $e(i)$, and then allocates the excess capacity $\mathcal{E}=\mathcal{C}-\sum_{1<i<n} e(i) \geq 0$ in the ratio of their unmet demands $\delta(i)=d(\bar{i})-e(i)$. Demand-limited VMs have $e(i)=d(i)$ and hence do not receive additional allocation, while the rest receive a proportional amount of the excess capacity.

The allocation made by shareBPX will satisfy the properties described in Section 2. The harmonious property ensures that no VM will get more than its demand unless all the VMs do so; otherwise the algorithm would waste needed capacity. Freedom from share and demand anomalies are also satisfied. Note it is not obvious that these properties (particularly freedom from share anomaly) hold, since the allocation is made up of two parts: a share-sensitive part that allocates more capacity to VMs with higher shares and a demandsensitive part that allocates capacity in proportion to unmet demand. For two VMs with the same demand, a higher share VM will have less unmet demand compared to a lower share VM with the same demand. Hence, the former will be allocated less capacity than the latter in the demand-sensitive allocation. One needs to ensure that the demand-sensitive allocation does not overwhelm the share-sensitive part to avoid share anomalies. We show this property in Lemma 5.

If we apply shareBPX to the VMs of Example 2, it will result in the allocations shown previously in Table 2 . We end the section by formally showing that shareBPX satisfies the allocation properties.

LEMMA 2. The allocations of shareBPX are harmonious.

Proof: We will prove the harmonious condition by contradiction. Assume, to the contrary, that there is a pair of VMs $i, j$ such that $a(i) \geq d(i)$ and $a(j)<d(j)$. The allocation 


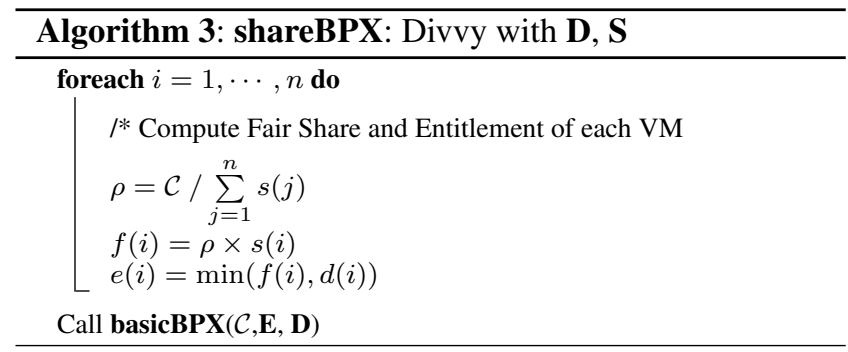

made to $\mathrm{VM}(i)$ by shareBPX is:

$$
\begin{aligned}
& a(i)=e(i)+\delta(i) / \sum_{k=1}^{n} \delta(k) \times \mathcal{E} \geq d(i) \\
& \delta(i) / \sum_{k=1}^{n} \delta(k) \times \mathcal{E} \geq d(i)-e(i)=\delta(i) \\
& \mathcal{E} \geq \sum_{k=1}^{n} \delta(k)
\end{aligned}
$$

Writing a similar inequality for $a(j)$ results in:

$a(j)=e(j)+\delta(j) / \sum_{k=1}^{n} \delta(k) \times \mathcal{E}<d(j)$

$\mathcal{E}<\sum_{k=1}^{n} \delta(k)$

This proves the contradiction.

LEMMA 3. The allocation made by shareBPX is given by:

$$
\begin{aligned}
& a(i)=\left\{\begin{array}{l}
d(i) \\
\alpha s(i)+\beta d(i)
\end{array} \quad \begin{array}{l}
\text { if } V M(i) \text { is demand limited } \\
\text { otherwise }
\end{array}\right. \\
& \text { where } \alpha=\rho(1-\beta), \beta=\frac{\mathcal{E}}{\sum_{k=1}^{n} \delta(k)}, \text { and } \mathcal{E}=\mathcal{C}-\sum_{k=1}^{n} e(k) .
\end{aligned}
$$

Proof: The allocation to $\operatorname{VM}(i)$ can be written as: $a(i)=$ $e(i)+\delta(i) / \sum_{k=1}^{n} \delta(k) \times \mathcal{E}$ where $\delta(k)=d(k)-e(k)$. For a demand-limited VM, $e(i)=d(i)$ and $\delta(i)=0$; hence the allocation $a(i)=d(i)$. For a VM that is not demand limited, $e(i)=f(i)=s(i) \times \rho$. Hence, $a(i)=e(i)+$ $\left((d(i)-e(i)) / \sum_{k=1}^{n} \delta(k)\right) \times \mathcal{E}=e(i)+\beta(d(i)-e(i))=$ $e(i)(1-\beta)+\beta d(i)=s(i) \rho(1-\beta)+\beta d(i)$. Hence we get: $a(i)=\alpha s(i)+\beta d(i)$.

LEMMA 4. Allocations made by shareBPX are free of demand anomalies.

Proof: Suppose VMs $i$ and $j$ have the same share, $s(i)=$ $s(j)$. If either or both $i$ and $j$ are demand-limited then the result is easy to see. If neither is demand limited, then from Lemma 3, $a(i)=\alpha s(i)+\beta d(i)$ and $a(j)=\alpha s(j)+\beta d(j)$. Hence $a(i)-a(j)=\beta(d(i)-d(j))$. Since $\beta>0$, it follows that $a(i)>a(j)$ if and only if $d(i)>d(j)$. In fact the difference in the allocations of two equal-share VMs is proportional to the difference in their demands.

LEMMA 5. Allocations made by shareBPX are free of share anomalies if $\mathcal{C} \leq \sum_{i=1}^{n} d(i)$.

Proof: If VMS $i$ and $j$ have the same demand, $d(i)=$ $d(j)$. Once again, the case when neither is demand-limited needs to be shown. Using Lemma 3 , we get $a(i)-a(j)=$ $\alpha(s(i)-s(j))$. To be free of share anomalies we require that $\alpha>0$ in the region of interest. Since $\alpha=\rho(1-\beta)$ and $\rho>0$ we require $\beta<1$.
Now $\mathcal{C}=\sum_{k=1}^{n} f(k)$ by the definition of fair share. Also, $\beta=\mathcal{E} / \sum_{k=1}^{n} \delta(k)$, and $\mathcal{E}=\mathcal{C}-\sum_{k=1}^{n} e(k)$.

Let $D$ and $N$ denote the set of demand-limited VMs and non-demand-limited VMs respectively.

$\mathcal{E}=\sum_{k=1}^{n} f(k)-\sum_{k=1}^{n} e(k)=\sum_{k=1}^{n} f(k)-\left(\sum_{k \in N} f(k)+\right.$ $\left.\sum_{k \in D} d(k)\right)=\sum_{k \in D}(f(k)-d(k))$.

Now $\sum_{k=1}^{n} \delta(k)=\sum_{k \in N} \delta(k)=\sum_{k \in N}(d(k)-f(k))$.

For $\beta<1$, we require that $\mathcal{E}<\sum_{k=1}^{n} \delta(k)$.

This is true if $\sum_{k \in D} f(k)+\sum_{k \in N} f(k)<\sum_{k \in D} d(k)+\sum_{k \in N} d(k)$, or $\mathcal{C} \leq \sum_{k=1}^{n} d(k)$.

This is true by hypothesis.

\section{System Level Implementation of BPX}

In this section we present the details of implementing BPX as a prototype in our cluster management solution called DRS [13]. DRS runs as part of VMware's virtual center software that can manages hundreds of ESX servers in a virtual environment. An administrator creates a cluster of up to 32 hosts and in a cloud environment there may be tens to hundreds of clusters, each managed by an instance of DRS. Figure 3 shows an overview of the system. Figure 4 shows an example distribution of tenants across the clusters. BPX allows a tenant's VMs to be spread across multiple clusters, and each cluster can have VMs from multiple tenants.

DRS offers three controls for each VM: minimum reservation (r), maximum limit (1) and shares (s) for both $\mathrm{CPU}$ and memory resources. We first extend the BPX algorithm (previously described using only shares and demands) to incorporate VM reservations and limits as well. We then describe how the implementation integrates into the existing DRS infrastructure to provide a scalable resource allocation mechanism based on the BPX algorithm.

BPX has two properties that help the algorithm to scale. First it summarizes information by adding together VMlevel statistics. Only this aggregated information is needed for allocation, minimizing the information exchanged between participating nodes, and simplifying distributed implementation. Secondly, the allocations can be computed at any granularity (VM, server, cluster) simply by knowing the total aggregated value of the statistic and the aggregated value for the VMs in the subtree.

Periodically, (every 1 minute in our prototype), each DRS cluster publishes a set of aggregated values for each of its tenants to a distributed key-value store that can be accessed by all DRS clusters. Each cluster only has to publish one fixed size record (of six aggregated statistics) per tenant. This leads to huge reduction in overall communication from one record per VM to one per tenant, which is a much smaller number.

The published record includes the sum of the reservations, limits, shares, demand, and two entitlement values (see Algorithm 4) for VMs of the tenant. DRS also runs periodically (every 5 minutes in our prototype) to compute new allocations. It reads the aggregated stats published by the other clusters in the shared key-value store, and sums them to obtain the total aggregate values. It then uses BPX to divvy out 
the allocation. The VM allocations are then pushed to the ESX servers.

\subsection{BPX Divvying with $R, L$ and $S$ controls}

We now discuss the complete resource model and its BPX implementation. The tenant purchases capacity in the form of a total tenant reservation $\mathcal{R}$ and a total tenant limit $\mathcal{L}$. $\mathcal{R}$ will be divided among the active VMs, based on their shares and demands, to increase their run-time reservations over their static values. Similarly, the tenant specifies $\mathcal{L}$ as an overall cap on resource usage by all its VMs. This will be used to place run-time limits on individual VMs, which may be more conservative than their static values depending on their importance (share) and current demand.

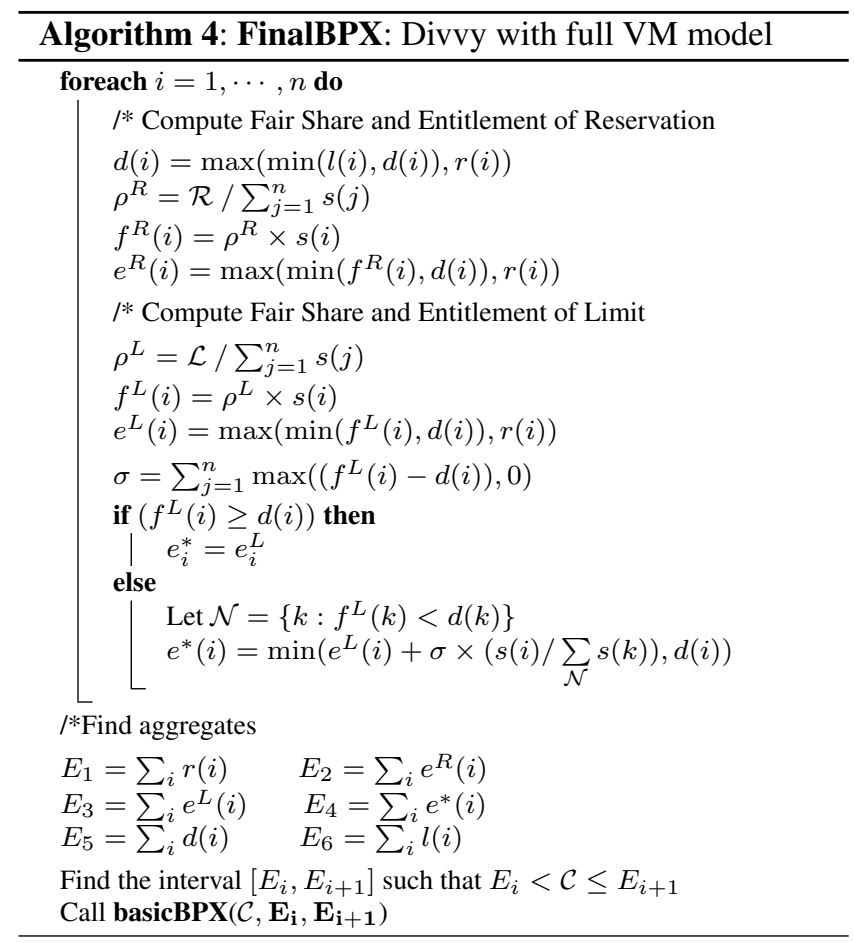

The extended BPX algorithm is described in Algorithm 4. $\operatorname{VM}(i)$ has two additional static parameters $r(i)$ (reservation) and $l(i)$ (limit), in addition to share $s(i)$ and demand $d(i)$. The demand $d(i)$ is first adjusted to lie between $r(i)$ and $l(i)$. The algorithm computes share-per-reservation, fair share, and entitlement (as in Algorithm 3) for capacity equal to both $\mathcal{R}$ and $\mathcal{L}$, as denoted by the superscript on the variables. It also computes an additional entitlement $e^{*}$ based on dividing the unallocated capacity generated by demandlimited VMs among the the non-demand limited VMs, in proportion to their shares.

Several aggregates $E_{1}$ to $E_{6}$ are identified in Algorithm 4. $E_{1}, E_{5}$ and $E_{6}$ are simply the sums of the reservations, demands, and limits of all the VMs. $E_{2}, E_{3}$ and $E_{4}$ are obtained by summing the different entitlements. To divvy a capacity $\mathcal{C}$ (either $\mathcal{R}$ or $\mathcal{L}$ ) we find which interval $\left[E_{i}, E_{i+1}\right]$ in which $\mathcal{C}$ lies, and use $E_{i}$ as the lower bound and $E_{i+1}$ as the upper bound for basicBPX. By using these multiple aggregates $E_{i}$, BPX narrows the portion of the capacity that is allocated based on demand. This allows it to more closely approach the allocation of the DDAP1 policy, while still maintaining its implementation advantages over the IDD algorithm. All of the allocation properties still hold with this enhancement.

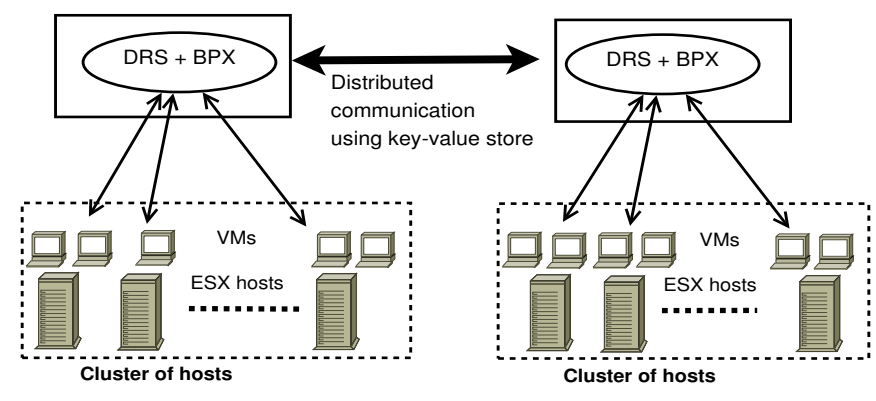

Figure 3. System Architecture with clusters and an instance of DRS managing each cluster. The data is shared between different clusters using a key-value store that spans the cloud environment.

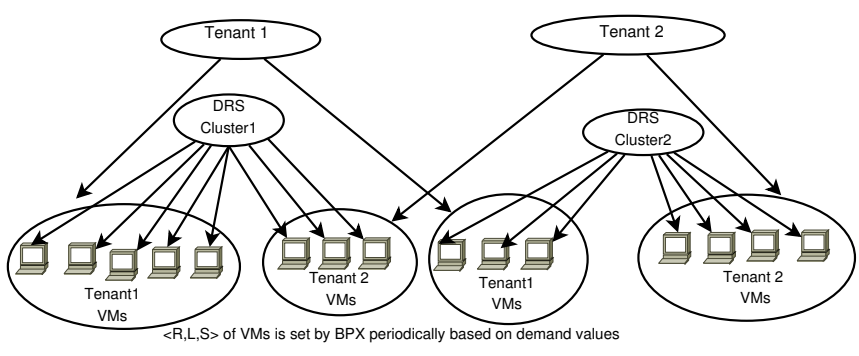

Figure 4. Tenant VMs are deployed across clusters and a cluster may host multiple tenants.

\subsubsection{Physical Capacity Limits}

Our goal is to flow resources to the set of VMs with high demand for each tenant. These are independent decisions made without explicit consideration of the underlying physical capacity. It is possible that the host running the VMs may not have enough capacity to handle all the new resource settings. DRS makes sure that each server can satisfy the resource requirements for the VMs running on it. If that is not the case, DRS recommends VM migrations to make sure that each server can handle these resource requirements.

In rare cases, an entire cluster can run short of resources to support the dynamic allocations. In that case, the longer term solution is to add more hardware to the cluster or migrate some VMs out of that cluster to another. We are working to implement the second solution, but in the interim we use the following approach. We reduce the per VM allocation based on its share without going below its static reservation. So a VM with high shares will loose less as compared to a VM with low shares.

\subsection{Additional Implementation Issues}

We address some of the issues that need to be handled in a complete implementation. 
Demand estimation: So far we have assumed VM demands as one of the inputs to our algorithm. In practice both CPU and memory demands are computed by the ESX hypervisor and published as a periodic statistic. A VM's CPU demand is computed as its actual CPU consumption, $C P U_{\text {used }}$, plus a scaled portion of $C P U_{\text {ready }}$, the time it was ready to execute, but queued due to contention:

$$
C P U_{\text {demand }}=C P U_{\text {used }}+\frac{C P U_{\text {run }} \times C P U_{\text {ready }}}{\left(C P U_{\text {run }}+C P U_{\text {sleep }}\right)}
$$

A VM's memory demand is computed by tracking a set of randomly-selected pages in the VM's physical address space, and computing how many of them are touched within a certain time interval [28]. For BPX, the above raw metrics are aggregated to get the demand over the entire divvying interval. We used 5 minute average demand in our prototype.

Shares Across Multiple Tenants: Since multiple tenants may be sharing the same underlying cluster, share values must be comparable across tenants. In addition, the share value of a VM must scale with number of vCPUs and memory size to avoid priority inversion when the same amount of resources is spread over more CPUs or memory. We therefore chose to use certain pre-configured values like high (2000), medium(1000) and low(500) per unit of resource.

Placement of VMs across clusters and hosts: Keeping VMs of a tenant within a small number of clusters (possibly one) is preferred. This can reduce stress on bi-section bandwidth and the complexity of BPX divvying in the system. The placement algorithm we use has a preference for a cluster where the tenant already has lot of VMs, and it selects a new cluster only if some constraint cannot be satisfied or if it will cause imbalance beyond a threshold for a resource.

Similarly if VMs from the same tenant are running on the same host, they can be grouped under a resource pool and the sum of their allocated reservation and limits can be set as the resource pool control instead of per VM control. This optimization allows the flow of resources within the host at sub-second granularity.

\section{Experimental Evaluation}

In this section we present results from our extensive evaluation of the BPX algorithm using both a simulated setup and real inventory using ESX hosts and virtual machines with variable workloads. The goal of our experiments is to show that BPX can do dynamic allocation of resources among the VMs of a tenant, similar to a centralized scheduler, although in a much more scalable manner. We also compared allocations of BPX with static allocation.

\subsection{Simulation Setup}

Our BPX prototype is implemented as part of DRS cluster manager. We used the same DRS simulator framework that is used for internal code development, to construct a cluster with a set of simulated ESX hosts and virtual machines. The same implementation of BPX and DRS is used in a simulator or a real inventory.

The simulator allows us to create different VM and host profiles with different configurations. A VM profile includes the number of virtual CPUs (vCPUs), configured CPU (in
$\mathrm{MHz}$ ) and configured memory size (in $\mathrm{MB}$ ), while a host profile includes the number of physical cores, $\mathrm{CPU}(\mathrm{MHz})$ per core, total memory size, idle power consumption etc. The simulator also supports arbitrary workload specifications for each VM over time and generates CPU and memory demand values based on the specification.

In addition to creating a cluster, the simulator also mimics the behavior of ESX CPU and memory schedulers and allocates resources to the VMs in a manner consistent with the behavior of ESX hosts. The simulator supports all the resource controls supported by the real ESX hosts, including reservation, limit and shares for each VM. Every 5 minutes, the simulator invokes the actual DRS code to figure out if any VM migrations are needed to meet VM demands. It also keeps track of how much resources are demanded by each VM and how much of those resources were given to the VM. This is used to calculate the key evaluation metric to figure out how well the demand based allocation works.

The main metric used to study how effectively resources are being allocated is a cumulative allocated percentage metric that is defined as follows:

$$
\sum_{t=1}^{T} \sum_{k=1}^{\text {AllVms }} \frac{\left(\text { Satisfied demand for } V M_{k} \text { at } t\right)}{\text { Cloud capacity at } t} * 100
$$

Here $T$ denotes the total simulation time. This metric is calculated separately for both CPU and memory. For CPU the metric captures the percentage of the total number of CPU cycles in the cluster (on all the hosts) over the period of the experiment, that were usefully spent. The memory metric denotes the similar percentage. The higher values are better.

We used multiple clusters in our experiments, each managed by DRS and ran BPX across clusters. We used BPX to allocate the overall resources bought by a tenant among its VMs. The BPX allocation is enforced by DRS. If in some cases, the sum of VM level settings become higher than the host capacity for CPU or memory, DRS would move VMs between hosts to make sure that VMs are able to meet their allocation. The overhead in terms of CPU and memory for a VM migration is also captured by the simulator which penalizes the cumulative metric based on time taken for migration and depends on how actively the memory is being dirtied.

\subsection{Single Tenant with Diverse Workloads}

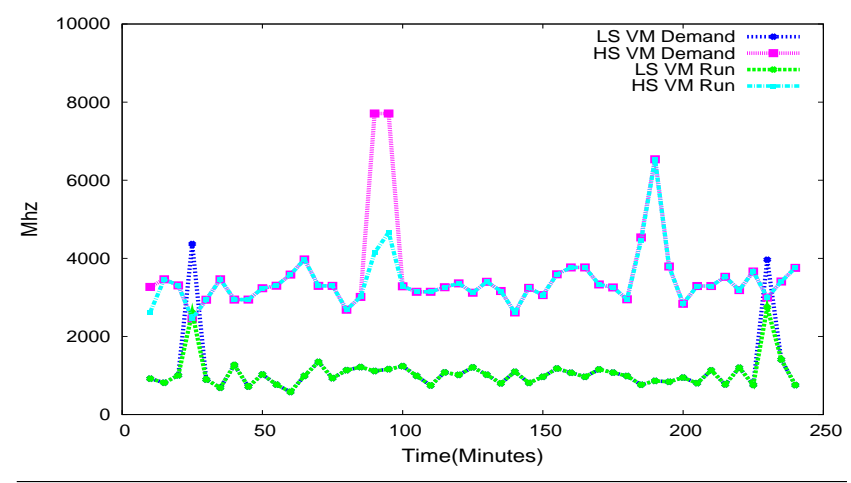

Figure 5. Demands and utilization of one high share VM and one low share VM. 


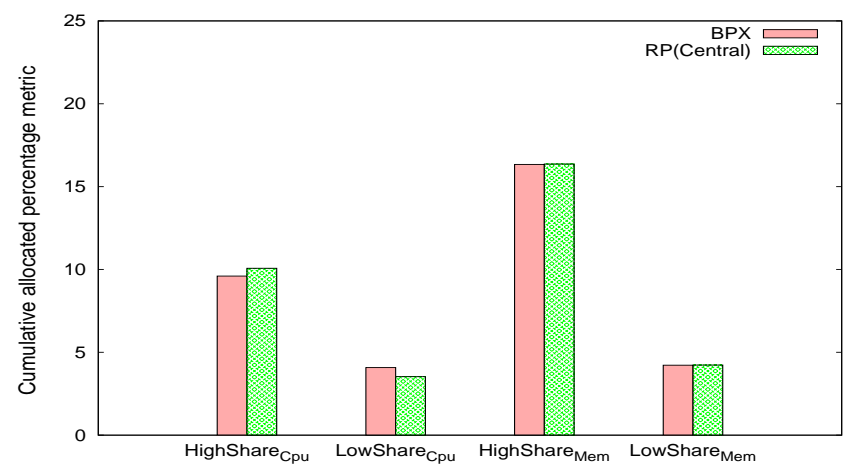

Figure 6. Cumulative BPX allocations compared with central divvy.

\begin{tabular}{|c|c|c|c|c|}
\hline VMType & Scenario1 & Scenario2 & Shares & Num VMs \\
\hline HS1 & High, Low & High, Low & High(2000) & 50 \\
\hline HS2 & High, Low & Low, High & High (2000) & 50 \\
\hline MS1 & High, Low & High, Low & Medium (1000) & 50 \\
\hline MS2 & High, Low & Low, High & Medium (1000) & 50 \\
\hline LS1 & High, Low & High, Low & Low (500) & 50 \\
\hline LS2 & High, Low & Low, High & Low (500) & 50 \\
\hline
\end{tabular}

Table 3. Tenant VM specifications. High is uniformly distributed between $3 \mathrm{GHz}$ and $4 \mathrm{GHz}$. Low is less than 400 $\mathrm{MHz}$.

In the first experiment, we tried out the scenario mentioned in section 2. There are 4 high share VMs (2000 shares each) and 4 low share VMs (500 shares each) belonging to a tenant. The high priority VM mostly consumes less than 4 $\mathrm{GHz}$ and sometimes spikes to $8 \mathrm{GHz}$. The low share VMs consume $1 \mathrm{GHz}$ and occasionally spike to $4 \mathrm{GHz}$. These eight VMs were run across 3 clusters and each with 6 hosts. Figure 5 shows the demands and utilization of one of the high share VMs and one of the low share VMs. Note that high share VM is able to get more resources when its demand is higher and low share VM is also able to get more resources during periods of low demand from other VMs.

To compare with a centralized solution, we ran all $8 \mathrm{VMs}$ in a single DRS cluster and measured the resources allocated to VMs by DRS. Figure 6 shows the comparison of the BPX and DRS allocations over 200 minutes. Overall the highshare VMs and low share VMs got similar allocation under both schemes. The small difference in allocation where high share VMs get slightly less in the case of BPX is because central scheduler does allocation perfectly in terms of shares whereas BPX does part of allocation based on shares and rest using the demand values.

\subsection{Large Setup with Coordinated Workloads}

In the next experiment, we evaluated how BPX performs in a larger setup with co-ordinated workload changes across a set of VMs. This is one of the adversarial workloads for BPX since the workload changes are not randomly distributed but synchronized. The setup consists of $300 \mathrm{VMs}$ belonging to a single tenant distributed over 120 hosts spread across 3 clusters. The VMs had a mix of high, medium and low shares that correspond to a ratio of $4: 2: 1$ respectively. We simulated a diurnal workload where the workload switches its state every one hour. The detailed VM specifications are mentioned in Table 3. When the VM is in state high, its workload value is randomly chosen uniformly between between $3 \mathrm{GHz}$ and $4 \mathrm{GHz}$. When the workload is in state low, it is uniformly between 0 and $400 \mathrm{MHz}$. The tenant is entitled to $160 \mathrm{GHz}$ of CPU and $160 \mathrm{~GB}$ of Memory.

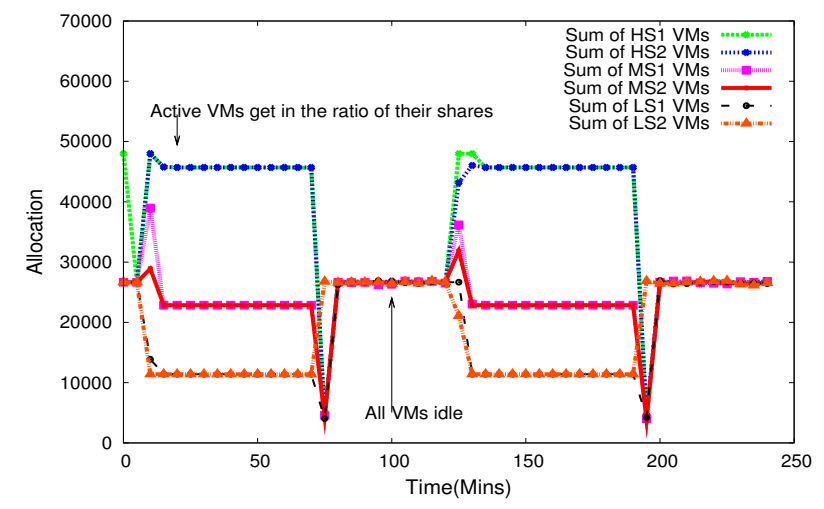

Figure 7. VM allocations are in the ratio of 1:2:4 when they are active.

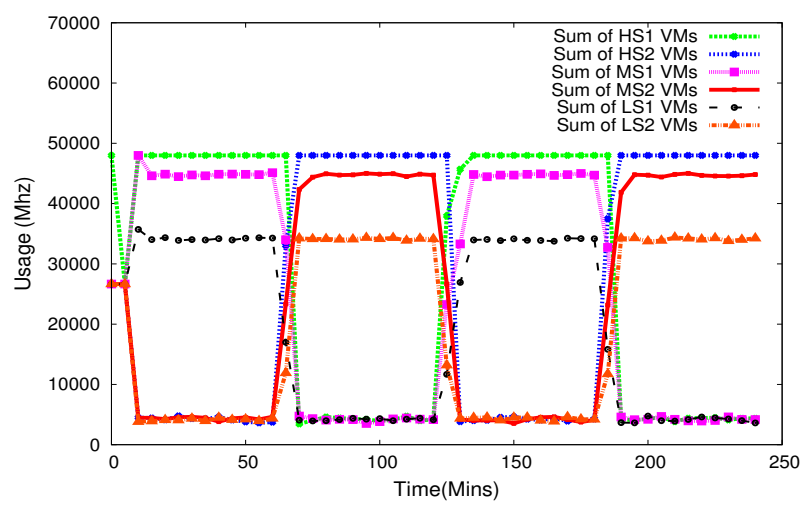

Figure 8. Time line of allocations to VMs as it becomes active and idle.

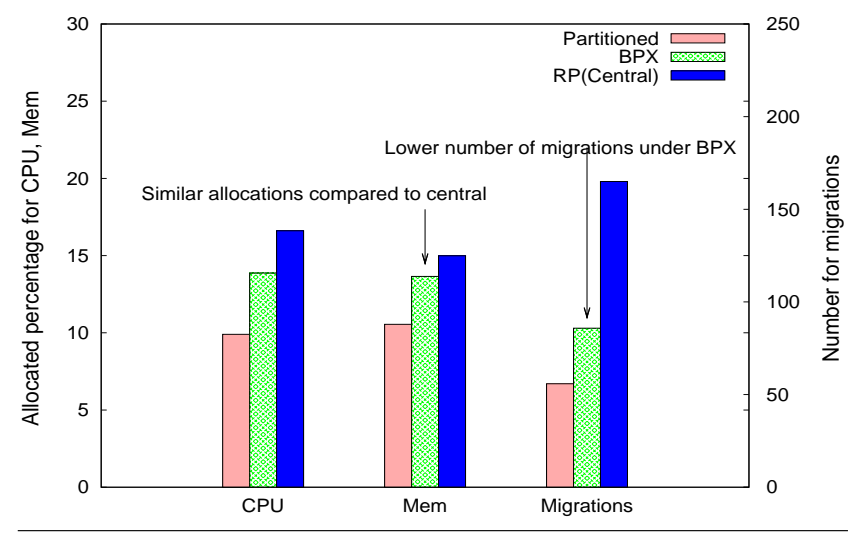

Figure 9. Comparison of BPX CPU and Memory cumulative allocations and number of migrations.

In the first scenario, all the VMs have a high workload for one hour followed by low workload for another one hour. 
Figure 7 shows the sum of allocations of all the $50 \mathrm{VMs}$ for each type. Initially note that the allocations of VMs are in proportion to their shares. At $t=60$ minutes, all the VMs abruptly become idle. Each cluster immediately sees its own VMs becoming idle, but takes few minutes to get stats from other clusters to realize other VMs have also gone idle and hence the allocation gets very low before bouncing back to a value that is greater than the demand. In this case all VMs get similar allocation due to lack of demand. At $t=120$ minutes, all the VMs become active at the same time and the system allocates the resources to the VMs again in the ratio of $1: 2: 4$ after each cluster grabs a little bit more for its VMs than it should. The key takeaway here is that when there is demand at high share VMs, they are able to get the resources and otherwise, all VMs get equal resources due to lack of demand.

In the next scenario, we tried a case where some VM sets become idle when the others become active. HS1 with high shares, MS1 with medium shares and LS1 with low shares become active together and HS2, MS2 and LS2 become active together. This is an interesting scenario for BPX as one set of high, medium and low share VMs become idle giving up their share to other VMs. Figure 8 shows the sum of allocation to all the $50 \mathrm{VMs}$ of each type. For their cumulative allocations, the high share VMs get more than the medium share VMs and the medium share VMs get more than the low share VMs. But the allocation is not exactly in the ratio of $1: 2: 4$ as the excess given up by the idle VMs is given to active VMs all three categories. But still high share VMs get more than medium share VMs and medium share VMs get more than low share VMs.

\subsection{Comparison with Other Allocations}

We also compared BPX with two other implementations: (1) Partitioned allocation with dynamic allocation within cluster and (2) centralized allocation for all VMs. In the first scheme, the overall capacity is statically divvied among clusters based on the number of VMs and their shares in the cluster. Within a cluster DRS then uses centralized algorithm to dynamically allocate the per cluster capacity every $5 \mathrm{~min}$ utes. This is already a very good contender since within a cluster we are doing dynamic allocation but not across clusters. We expect this to cause wastage of resources as some cluster might have idle capacity while other cluster might have more demand as compared to cluster capacity.

As second scheme, we tested a centralized divvy where we collect the demands of all the $300 \mathrm{VMs}$ in one location and do allocation per VM using the centralized algorithm mentioned in Section 3.2. This is equivalent to running the VMs in a single large cluster, which is how we implemented this mechanism. This solution obviously cannot scale, when we have large number of tenants or large number of VMs per tenant in a cloud environment. In all three schemes, DRS load balancing algorithm was run in each cluster to move VMs within the cluster when the allocation to VMs exceeded the host capacity.

Figure 9 shows the comparison among various schemes in terms of CPU and memory allocation metrics and the overall number of migrations that were done by DRS in or- der to handle host level contention. Centralized algorithm is very aggressive in terms of dynamic allocation and reallocating resources from VMs that are idle to the active ones. This is apparent from the large number of migrations that DRS did in case of centralized solution. The total allocation metric for CPU and Memory is also slightly better as compared to BPX because the central divvy can redistribute resources quickly and accurately.

With BPX, it takes some time for the allocations to happen and this causes the cumulative allocation metric to be slightly lower as compared to the centralized solution. The partitioned allocation scheme can re-distribute resources within a cluster but it could not re-distribute resources across clusters and ends up wasting resources that the tenant had bought. This causes the allocation metrics to be even lower than BPX. This experiment shows that BPX provides a good trade-off between a non-scalable centralized solution and demand-agnostic partitioned solution.

\subsection{Real Deployment of Hadoop and VDI VMs}

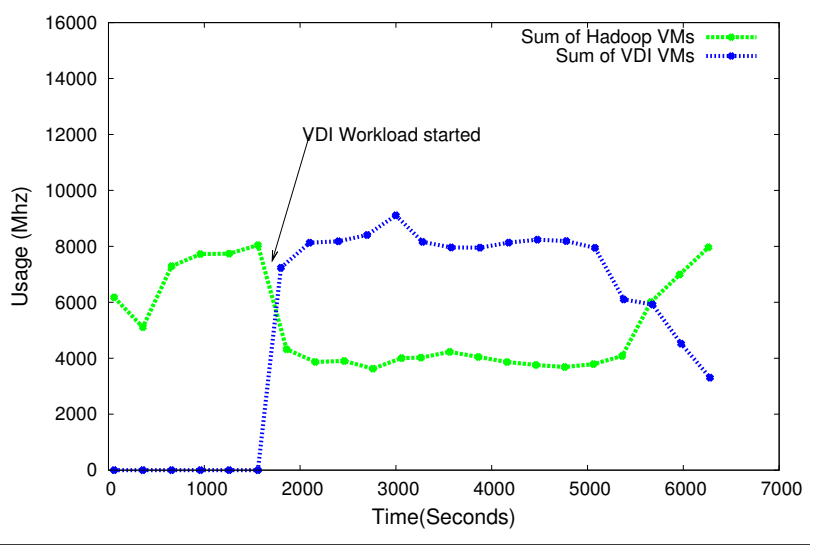

Figure 10. Sum of CPU usage of all Hadoop VMs and VDI VMs.

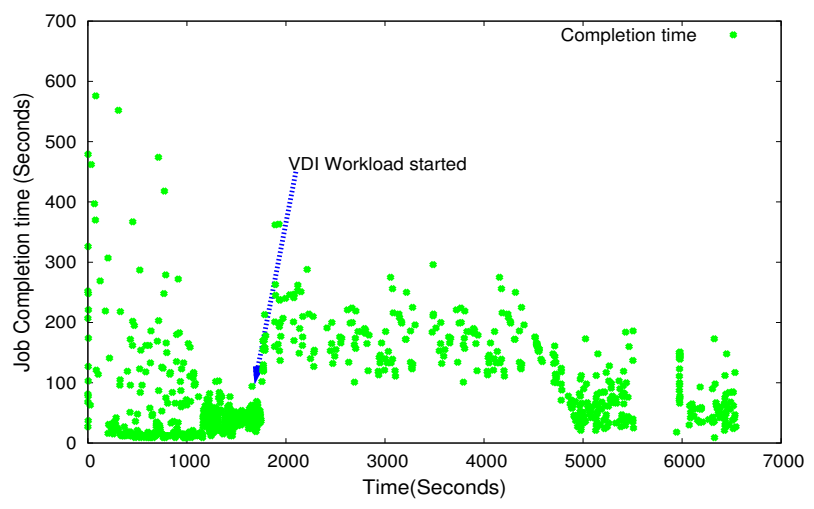

Figure 11. Hadoop job completion times over time of the experiment.

We also experimented with a real deployment with ESX hosts and VMs running various real workloads. We used two clusters with a total of 8 ESX hosts. The clusters were in two separate but nearby datacenters. Each cluster was 
managed by the DRS cluster manager software with our prototype BPX implementation. We ran Hadoop workload in one cluster and a virtual desktop interface (VDI) workload in another cluster.

We setup the controls to reflect a tenant who has bought $12 \mathrm{GHz}$ of CPU capacity and $12 \mathrm{~GB}$ of memory. Both the Hadoop workload and the VDI workload were run using this capacity. There were also other background workloads belonging to other tenants in the clusters running CPU intensive server workloads. We mainly focus on the tenant workload under study.

The Hadoop VMs were setup using Serengeti [26] and consisted of 10 worker VMs, one Master VM and one client VM. The Hadoop workload consisted of running the teragen and terasort programs. Teragen was run with one billion rows and 800 map jobs. Terasort was followed by 800 map and 500 reduce jobs.

The VDI workload consisted of 11 VDI(desktop VMs) and was run using the ViewPlanner benchmark from VMware. The workload consisted of a mix of desktop applications such as power point, zip, movie player etc. The ViewPlanner harness VMs were not part of the tenant and hence did not consume from the $12 \mathrm{GHz}$ available to tenant. The VDI VMs were configured with high shares as they are interactive workload while all the Hadoop VMs had low shares. The high shares were in the ratio of $4: 1$ compared to the low shares.

Initially the workload was started in the Hadoop cluster. Since the Hadoop VMs were the only VMs belonging to the tenant, they were able to use all of the purchased capacity upto their demand. The Hadoop workloads were consuming as much CPU as they had needed. At $t=1700$ seconds, the VDI workload was started, which powered on all the 11 VDI VMs in the second cluster and started the workload inside them. Since the tenant has only $12 \mathrm{GHz}$, and all the high share VDI VMs suddenly spiked up, the Hadoop VMs in the other cluster were throttled which caused their CPU consumption to go down and their job completion times to go up.

The VDI workload lasted for one hour and then VMs slowly became idle. As this happened, the Hadoop VMs were able to pick up the slack and they were given resources that were given up by the idle VDI VMs. Figure 10 shows the sum of CPU usage of all the Hadoop and the VDI VMs. Each point in the graph denotes the sum of the five minute moving average of the CPU usage of the VMs of each type. Note that the overall sum is always $12 \mathrm{GHz}$. Figure 11 shows the job completion time as measured by the Hadoop. The job completion time is plotted against time when the job started. Note that when the VDI workload was started, the job times of Hadoop increased as expected. Overall, these results show that BPX is able to allocate the overall bulk capacity purchased by a tenant among its VMs based on their demand and overall importance as denoted by shares. BPX also supports reservations and limit controls per VM, which we didn't specifically evaluate here.

\section{Related work}

Resource allocation and buying models in a public cloud environment is a fast evolving area. Here we focus on three broad areas: (1) capacity planning and demand prediction, (2) meeting application level SLAs and (3) dynamic resource distribution among VMs in a cloud.

Capacity planning and demand prediction are critical in deploying workloads in a cloud. Several approaches [9, 10, 34] have been proposed for demand prediction based on trace analysis, and using it for capacity estimation, and admission control. These approaches are complementary to BPX and can help a user estimate virtual capacity purchase decisions. Gmach et al. [11] proposed a global controller to create a self-managing pool of virtualized servers to satisfy time-varying service level objective and workload demands. This is similar to DRS cluster manager software, which is quite useful but may not scale well to a large number of servers. Wang et al. [32] studied how the cloud provider should allocate resources to different pricing schemes, such as PAYG and spot auctions so as to maximize revenue, while [18], proposed a new pricing scheme for selling network bandwidth reservations. This is an orthogonal problem to the issue solved by BPX.

Urgaonkar et al. [24] proposed an analytical model based on networks of queues to predict performance of multi-tiered applications for capacity planning. Casale et al. [7] showed how to incorporate burstiness into the analytical queuing network models. These approaches provide sound foundational basis for medium term or offline capacity estimation, in contrast to our adaptive approach based on measured performance. Several studies $[4,20]$ have proposed approaches for performance modeling and SLA based resource allocations for multi-tier applications. Providing QoS guarantees to applications while overcommitting the servers has been studied in [23, 29]. Such application-level approaches can be used in cases where a public cloud provider allows a user to set arbitrary resource controls on its VMs.

Mesos [6] proposes a two-level approach to allocate resources to frameworks like Hadoop and MPI that may share an underlying cluster of servers. Mesos (and related solutions) rely on OS-level abstractions like resource containers $[1,2,5]$, which are quite useful but not in common use in IaaS based public clouds, and are less flexible then the VM controls used here. R-Opus [8] provides an elegant framework to map application workload demands to various allocation priorities exposed by the workload manager. However both the application level QoS requirements and resource controls used by R-Opus are quite different from the ones used in public cloud environments.

The computational complexity of VM placement to improve consolidation ratios was studied in [25, 31]. In [14] a trace-based approach that takes in application level SLA's and determines which VMs are best run together on the same host was proposed to accommodate a number of concurrent workloads. In [22] a stochastic model to guide resource provisioning, and determine minimum capacity levels to meet service availability levels was proposed. In [30] a stochastic model that incorporates both diurnal variation and fast timescale burstiness was analyzed. The authors also studied the 
tradeoff between capacity and QoS for future performance requirements. In [21] resource usage patterns were analyzed using PCA techniques and shown to improve server consolidation ratios. In [33], they estimated resource requirements when workloads are moved from physical to a virtualized infrastructure.

Many of these solutions are complementary to BPX and can be used to determine the overall capacity that the tenant should buy. BPX can then handle runtime demand fluctuations by allocating the purchased capacity to the deserving VMs.

\section{Conclusions and Future Work}

In this paper, we studied the problem of selling cloud resources in bulk and providing the abstraction of physical resources to a customer. For example, a customer can buy 16 $\mathrm{GHz}$ of compute resources and deploy VMs with configured capacity of more than $16 \mathrm{GHz}$, thereby getting the benefit of statistical multiplexing among its workloads and doing overcommitment. We proposed two algorithms called IDD and BPX that dynamically allocate the overall capacity among VMs based on their demand, shares (i.e.importance), reservation and limit settings. We analyze BPX and show that it has better scalability and several desired properties that are needed in a shared environment.

We implemented BPX as part of VMware's management software and show that it can re-allocate resource in the order of minutes among a set of VMs running across different hosts and racks in a datacenter. We think that this model would enable the customers to get even more out of a public cloud deployment than buying VMs with fixed capacity and paying for their configured size.

\section{References}

[1] Linux containers (LXC) overview document. http://lxc. sourceforge.net/lxc.html.

[2] Solaris Resource Management. http://docs.sun.com/app/ docs/doc/817-1592.

[3] B. Agrawal, L. Spracklen, S. Satnur, and R.Bidarkar. Vmware view 5.0 performance and best practices. 2011. http://www.vmware.com/files/pdf/view/VMware-View-PerformanceStudy-Best-Practices-Technical-White-Paper.pdf.

[4] D. Ardagna, M. Trubian, and L. Zhang. SLA based resource allocation policies in autonomic environments. J. Parallel Distrib. Comput., 67 (3):259-270, 2007.

[5] G. Banga, P. Druschel, and J. C. Mogul. Resource containers: a new facility for resource management in server systems. In OSDI ' 99 .

[6] H. Benjamin and et. al. Mesos: a platform for fine-grained resource sharing in the data center. In NSDI' 11.

[7] G. Casale, N. Mi, L. Cherkasova, and E. Smirni. How to parameterize models with bursty workloads. SIGMETRICS Perform. Eval. Rev., 36 (2):38-44, 2008.

[8] L. Cherkasova and J. A. Rolia. R-opus: A composite framework for application performability and qos in shared resource pools. In $D S N$, pages 526-535, 2006.

[9] D. Gmach, J. Rolia, L. Cherkasova, and A. Kemper. Capacity management and demand prediction for next generation data centers. In ICWS, pages 43-50, 2007.

[10] D. Gmach, J. Rolia, L. Cherkasova, G. Belrose, T. Turicchi, and A. Kemper. An integrated approach to resource pool management: Policies, efficiency and quality metrics. In DSN, pages 326-335, 2008.
[11] D. Gmach, J. Rolia, and L. Cherkasova. Satisfying service level objectices in a self-managing resource pool. In SASO, 2009.

[12] D. Gmach, J. Rolia, and L. Cherkasova. Selling t-shirts and time shares in the cloud. In CCGRID, pages 539-546, 2012.

[13] A. Gulati, A. Holler, M. Ji, G. Shanmuganathan, C. Waldspurger, and X. Zhu. VMware Distributed Resource Management: Design, Implementation, and Lessons Learned. In VMware Technical Journal, March 2012.

[14] X. Meng, C. Isci, J. O. Kephart, L. Zhang, E. Bouillet, and D. E. Pendarakis. Efficient resource provisioning in compute clouds via vm multiplexing. In $I C A C$, pages 11-20, 2010.

[15] Microsoft, Inc. Microsoft Hyper-V Server. 2012. http://www . microsoft.com/en-us/server-cloud/hyper-v-server/ default.aspx.

[16] Nebula, Inc. 2012. http://www.nebula.com/.

[17] Nimbula, Inc. 2012. http://www. nimbula.com/.

[18] D. Niu, C. Feng, and B. Li. Pricing cloud bandwidth reservations under demand uncertainty. In SIGMETRICS, pages 151-162, 2012.

[19] L. Spracklen, B. Agrawal, R.Bidarkar, and H. Sivaraman. Comprehensive user experience monitoring. March 2011. VMware Technical Journal.

[20] C. Stewart and K. Shen. Performance modeling and system management for multi-component online services. In NSDI, 2005.

[21] J. Tan, P. Dube, X. Meng, and L. Zhang. Exploiting resource usage patterns for better utilization prediction. In ICDCS Workshops, pages 14-19, 2011

[22] Y. Tan, Y. Lu, and C. H. Xia. Provisioning for large scale cloud computing services. In SIGMETRICS, pages 407-408, 2012.

[23] B. Urgaonkar, P. J. Shenoy, and T. Roscoe. Resource overbooking and application profiling in shared hosting platforms. In OSDI, 2002.

[24] B. Urgaonkar, G. Pacifici, P. J. Shenoy, M. Spreitzer, and A. N. Tantawi. An analytical model for multi-tier internet services and its applications. In SIGMETRICS, pages 291-302, 2005.

[25] B. Urgaonkar, A. L. Rosenberg, and P. J. Shenoy. Application placement on a cluster of servers. Int. J. Found. Comput. Sci., 18(5), 2007.

[26] VMware Big Data team. 2012. http://www . vmware.com/hadoop/ serengeti.html.

[27] VMware, Inc. VMware vCloud Suite. 2012. http: //www . vmware.com/products/datacenter-virtualization/ vcloud-suite/overview.html.

[28] C. A. Waldspurger. Memory Resource Management in VMware ESX Server. In USENIX OSDI '02.

[29] H. Wang, K. Doshi, and P. Varman. Nested QoS: Adaptive burst decomposition for SLO guarantees in virtualized servers. Intel Technology Journal, 16:156-181, June 2012. ISSN 0018-9340.

[30] K. Wang, M. Lin, F. Ciucu, A. Wierman, and C. Lin. Characterizing the impact of the workload on the value of dynamic resizing in data centers. In SIGMETRICS, pages 405-406, 2012.

[31] M. Wang, X. Meng, and L. Zhang. Consolidating virtual machines with dynamic bandwidth demand in data centers. In INFOCOM, 2011.

[32] W. Wang, B. Li, and B. Liang. Towards optimal capacity segmentation with hybrid cloud pricing. In ICDCS, pages 425-434, 2012.

[33] T. Wood, L. Cherkasova, K. M. Ozonat, and P. J. Shenoy. Profiling and modeling resource usage of virtualized applications. In Middleware, pages 366-387, 2008.

[34] Q. Zhang, L. Cherkasova, G. Mathews, W. Greene, and E. Smirni. R-capriccio: A capacity planning and anomaly detection tool for enterprise services with live workloads. In Middleware, 2007. 